Minireview

\title{
Virus-mediated mRNA decay by hyperadenylation
}

\author{
Kevin J Sokoloski, Emily L Chaskey and Jeffrey Wilusz
}

Address: Department of Microbiology, Immunology and Pathology, Colorado State University, Fort Collins, CO 80523, USA.

Correspondence: Jeffrey Wilusz. Email: jeffrey.wilusz@colostate.edu

\begin{abstract}
Degradation of cellular mRNAs during Kaposi's sarcoma-associated herpesvirus infection is associated with hyperadenylation of transcripts and a relocalization of cytoplasmic poly(A)-binding proteins to the nucleus.
\end{abstract}

The cellular machinery for RNA decay plays a major role in regulating gene expression and as a mechanism for RNA quality control [1]. Increasing evidence suggests that viruses have evolved ways of interfacing with the cellular RNA decay machinery that aid their survival and replication. First, viral transcripts must avoid degradation if they are to be effectively translated. Second, viruses often induce the degradation of cellular mRNAs, which gives their own transcripts a competitive edge for access to the cellular translation machinery. The mechanisms underlying these strategies are currently being elucidated. In addition to providing a clearer understanding of virus-host interactions, the mechanisms used by viruses to usurp the cellular RNA decay machinery may also provide insight into innate cellular mechanisms. This point is well illustrated in a recent paper in PLoS Biology by Yeon Lee and Britt Glaunsinger [2] on a novel RNA decay mechanism induced by Kaposi's sarcoma-associated herpesvirus (KSHV). Kaposi's sarcoma is the most common tumor in people with AIDS and results from chronic infection with the virus. However, like other herpesviruses, KSHV causes a lytic infection when reactivated and during this phase shuts off host-cell functions by inducing a global destruction of mRNA.

\section{KSHV-encoded SOX protein induces mRNA decay}

KSHV initiates global decay of cellular mRNAs via expression of the virus-encoded ShutOff and Exonuclease (SOX) protein [3]. Unlike the virion shutoff protein (VHS) of the related herpes simplex virus [4], SOX itself does not possess any demonstrable nuclease activity [5], and so how it induces mRNA decay is of considerable interest. In addition, bioinformatic analyses fail to identify any protein-protein interaction domain that would provide a clue to possible co-effectors of SOX-induced mRNA degradation. Thus, Lee and Glaunsinger [2] had relatively little to guide them as they set out to define the mechanism of SOX-induced RNA decay.

Through a careful analysis of mRNA modifications, localization, and RNA-binding proteins during SOX-induced mRNA degradation, Lee and Glaunsinger made four key observations using a series of transfections and viral infections in human 293 T and TIME (telomerase-immortalized microvascular endothelial) cells. First, they documented a clear increase in the size of the poly(A) tail of target RNAs in the presence of SOX that correlated with a decrease in the relative stability of the transcripts. Presumably this is due to the addition of adenosines, although other nucleotides cannot formally be ruled out [6]. Second, PAPII, the major poly(A) polymerase in the cell that is responsible for the initial mRNA polyadenylation event, was required for this hyperadenylation. This suggests that the PAPII is involved in the hyperadenylation, although it is not entirely clear whether its role is simply to provide the poly(A) tail to be extended or if it is directly responsible for adding the extra 3' nucleotides. Another protein that influences the primary polyadenylation event, the nuclear poly(A)-binding protein PABPN1 [7], is also required for SOX-mediated mRNA hyperadenylation and decay. Third, there was a dramatic increase in poly $(\mathrm{A})^{+}$ RNAs in the nucleus, suggesting that the hyperadenylation occurred on many different mRNAs and that an mRNAtrafficking pathway was probably being affected. Fourth, in the presence of SOX, the cytoplasmic poly(A)-binding protein PABPC1 was dramatically relocalized to the nucleus. A similar relocalization of PABPC1 to the nucleus has also been observed in patient-derived KSHV-infected cell lines [8]. Movement of PABPC1 to the nucleus was directly correlated with the ability of SOX protein to induce decay of cytoplasmic RNAs. Furthermore, knockdowns of PABPC1 by RNA interference (RNAi) reduced the ability of SOX to induce RNA turnover. Finally, reporter mRNAs (made using ribozyme technology) that lacked a 3' poly(A) were immune to SOX-mediated RNA degradation, directly correlating hyperadenylation with SOX-mediated decay. Interestingly, histone mRNAs that naturally lack a poly(A) tail can still be degraded in a SOX-dependent fashion even though they are not hyperadenylated. Thus, whereas the bulk of mRNA decay mediated by SOX involves 
hyperadenylation and PABPC1 relocalization, alternative degradation pathways appear to exist.

Because hyperadenylation of RNAs has been associated with nuclear surveillance for RNA quality in yeast $[9,10]$, and to a lesser extent in mammals [11,12], an attractive hypothesis is that SOX is causing the cell's quality control/ RNA surveillance machinery to degrade normal mRNAs in some fashion, perhaps by reorganizing the structure of messenger RNA ribonucleoprotein (mRNP) particles. Although this idea is consistent with the PABPC1 relocalization to the nucleus, it should be emphasized that it is currently unclear whether this relocalization is a cause, or a consequence, of SOX-induced RNA degradation. The SOX protein does not possess known interaction domains for poly(A)-binding proteins (for example, PAM2 [13]), nor do SOX and PABPC1 co-immunoprecipitate. Thus, SOX is likely to modulate PABPC1 localization via an indirect mechanism.

\section{Curtailing the actions of poly(A)-binding proteins is a common viral strategy}

Poly(A)-binding proteins have a multitude of functions in the cell, including the stimulation of polyadenylation, the nuclear export of mature mRNAs, regulation of translation efficiency and an influence on mRNA decay [14]. They therefore make an attractive target for viruses, as interfering with poly(A)-binding function would have a ripple effect on gene expression throughout the cell. In fact, as outlined in Figure 1, numerous RNA viruses, including picornaviruses, caliciviruses, HIV, rotavirus, rubella virus and now KSHV, have evolved strategies to interfere with this function. These include the cleavage, subcellular relocalization and binding/sequestration of poly(A)binding proteins, as well as the inclusion of binding sites for poly(A)-binding proteins in the viral genome that are not adenosine tracts - all of which could interfere with the normal function of poly(A)-binding proteins. In the absence of functional PABPC1, viruses would naturally have to develop a mechanism for maintaining the stability and translatability of their mRNAs; this is achieved in some viruses by the presence of internal ribosome entry sites (IRES) [15]. Curiously, KSHV appears to lack IRES elements.

\section{Unanswered questions and future directions}

KSHV induces the decay of approximately $95 \%$ of the cellular mRNA during infection, and it is unknown how sufficient levels of its own viral mRNAs escape SOXinduced mRNA decay to support a productive infection. Bioinformatic analysis of herpesvirus mRNAs fails to reveal any of the cis-elements that commonly mediate SOX resistance. There are at least three ways that KSHV mRNAs could selectively escape SOX-mediated RNA decay. First, they might encode a cis-acting element or bind to a transacting factor that stabilizes the poly(A) tail and thus prevents hyperadenylation and/or degradation. The nuclear

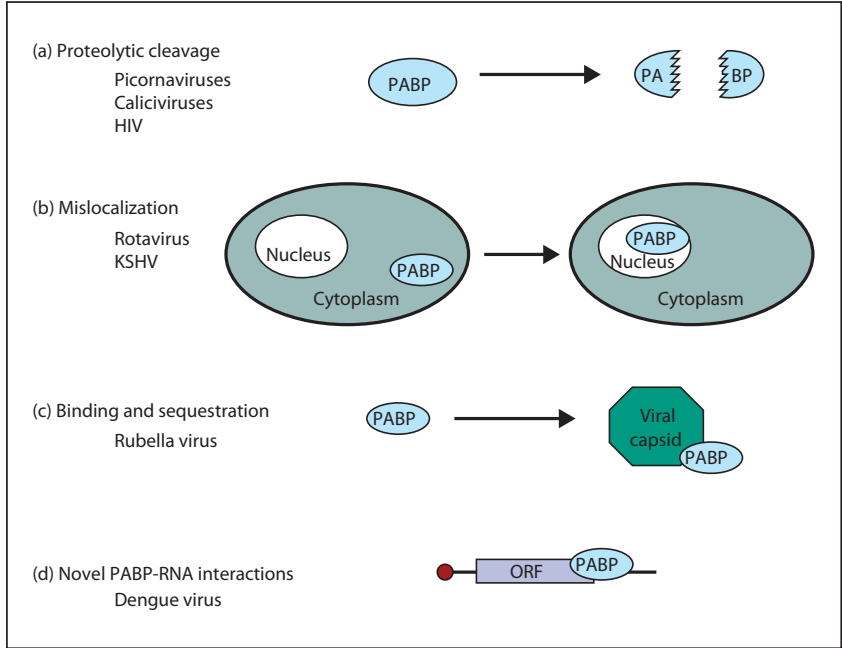

Figure 1

PABPC1 is a common target for viral perturbation of cellular processes. RNA viruses have developed a variety of strategies to interfere with or usurp the cytoplasmic poly(A)-binding protein PABPC1. This interference generally shuts down the translation of host-cell mRNAs as well as potentially exposing them to rapid degradation by the RNA decay machinery. (a) A variety of picornaviruses [22], caliciviruses [23] and HIV [24] encode proteases (for example, poliovirus $2 \mathrm{~A}$ ) that specifically cleave PABPC1. (b) The rotavirus nsp3 protein [25], as well as the KSHV SOX protein [2], relocalizes PABPC1 to the nucleus. Interestingly, unlike SOX, nsp3-induced relocalization does not appear to result in increased mRNA decay. (c) Rubella virus capsid protein specifically binds to PABPC1, sequestering the protein and presumably preventing its binding to cellular mRNAs [26]. (d) Despite the absence of a poly $(A)$ tract, sequences in the 3' untranslated region of dengue virus can specifically bind PABPC1 [27] and recruit it for use by viral mRNAs. ORF, open reading frame.

expression and retention element (ENE) in the KSHV PAN RNA [16] is a known example of a cis-acting element that is essential for the nuclear accumulation of this RNA. A second possibility is that whereas polyadenylation appears to deposit the protein nucleophosmin near the 3' end of cellular mRNAs [17], perhaps different proteins (with different downstream effects on mRNA fate) are deposited as a result of polyadenylation on virally encoded signals. Third, because of differences in RNA elements or mRNP structures, viral and cellular mRNAs may interact differently with components of the nuclear export machinery, thereby altering mRNA fate. Interestingly, KSHV encodes orf57, a protein required for lytic infection that stabilizes and exports the intron-less viral mRNAs from the nucleus [18]. If a failure to export mRNAs from the nucleus is related to SOX-mediated shutoff of cellular RNAs, could this factor be responsible for the SOX resistance of viral RNAs? Undoubtedly, further research will be focused on determining the resistance of viral transcripts to the mislocalization of PABPC1 and the induced mRNA decay. 
The SOX-mediated hyperadenylation of mRNA raises several interesting questions. First, what proteins are directly responsible for the 3'-end mRNA tailing during KSHV infection? Although PAPII is a strong candidate, the potential roles of numerous cellular non-canonical poly(A/U) polymerases [6] has not been tested. Furthermore, tailing mRNAs with uridines rather than adenosines has been shown to activate the decay of mammalian histone mRNAs [19] and certain transcripts in Schizosaccharomyces pombe [20]. Second, although it is assumed that hyperadenylation probably sets up a platform for exonucleases (as in the TRAMP pathway for RNA decay [21]), this needs to be formally demonstrated in SOX-mediated decay. The identity of the mRNA decay/ surveillance pathway that is being usurped by SOX is, therefore, of great interest. Third, it is unclear whether the poly(A)+ mRNAs that are sequestered in the nucleus as a consequence of SOX expression are cytoplasmic transcripts relocalized via PABPC1 or whether they are nascent mRNAs that accumulate as a result of a SOX-induced block in nuclear mRNA export. Answers to these and other questions will assuredly provide greater insight into our understanding of herpesvirus biology and cellular mRNA decay/surveillance mechanisms.

\section{Acknowledgements}

Studies on viral mRNA decay in the Wilusz laboratory are supported by NIH grant Al63434.

\section{References}

1. Garneau NL, Wilusz J, Wilusz CJ: The highways and byways of mRNA decay. Nat Rev Mol Cell Biol 2007, 8:113-126.

2. Lee YJ, Glaunsinger BA: Aberrant herpesvirus-induced polyadenylation correlates with cellular messenger RNA destruction. PLoS Biol 2009, 7:e1000107.

3. Glaunsinger BA, Ganem D: Lytic KSHV infection inhibits host gene expression by accelerating global mRNA turnover. Mol Cell 2004, 13:713-723.

4. Korom M, Wylie KM, Morrison LA: Selective ablation of virion host shutoff protein RNase activity attenuates herpes simplex virus 2 in mice. $J$ Virol 2008, 82:3642-3653.

5. Glaunsinger B, Chavez L, Ganem D: The exonuclease and host shutoff functions of the SOX protein of Kaposi's sarcoma-associated herpesvirus are genetically separable. $J$ Virol 2005, 79:7396-7401.

6. Kwak JE, Wickens M: A family of poly(U) polymerases. RNA 2007, 13:860-867.

7. Küehn U, Güendel M, Knoth $A$, Kerwitz $Y$, Rüedel S, Wahle E: Poly(A) tail length is controlled by the nuclear poly(A) binding protein regulating the interaction between poly $(A)$ polymerase and the cleavage and polyadenylation specificity factor. $J$ Biol Chem, in press.

8. Arias C, Walsh D, Harbell J, Wilson AC, Mohr I: Activation of host translational control pathways by a viral developmental switch. PLoS Pathog 2009, 5:e1000334.

9. Houseley J, Tollervey D: The nuclear RNA surveillance machinery: the link between ncRNAs and genome structure in budding yeast? Biochim Biophys Acta 2008, 1779: 239-246.

10. Anderson JT, Wang $X$ : Nuclear RNA surveillance: no sign of substrates tailing off. Crit Rev Biochem Mol Biol 2009, 44:16-24.
11. Slomovic, S, Laufer, D, Geiger, D, Schuster, G: Polyadenylation of ribosomal RNA in human cells. Nucleic Acids Res 2006, 34:2966-2975.

12. West S, Gromak N, Norbury CJ, Proudfoot NJ: Adenylation and exosome-mediated degradation of cotranscriptionally cleaved pre-messenger RNA in human cells. Mol Cell 2006, 21:437-443.

13. Roy G, De Crescenzo G, Khaleghpour K, Kahvejian A, O'Connor-McCourt M, Sonenberg N: Paip1 interacts with poly(A) binding protein through two independent binding motifs. Mol Cell Biol 2002, 22:3769-3782.

14. Mangus DA, Evans MC, Jacobson A: Poly(A)-binding proteins: multifunctional scaffolds for the post-transcriptional control of gene expression. Genome Biol 2003, 4:223.

15. Kieft JS: Viral IRES RNA structures and ribosome interactions. Trends Biochem Sci 2008, 33:274-283.

16. Conrad NK, Mili S, Marshall EL, Shu MD, Steitz JA: Identification of a rapid mammalian deadenylation-dependent decay pathway and its inhibition by a viral RNA element. Mol Cell 2006, 24:943-953.

17. Palaniswamy $\mathrm{V}$, Moraes $\mathrm{KC}$, Wilusz $\mathrm{CJ}$, Wilusz $\mathrm{J}$ : Nucleophosmin is selectively deposited on mRNA during polyadenylation. Nat Struct Mol Biol 2006, 13:429-435.

18. Boyne JR, Colgan KJ, Whitehouse A: Recruitment of the complete hTREX complex is required for Kaposi's sarcoma-associated herpesvirus intronless mRNA nuclear export and virus replication. PLoS Pathog 2008, 4:e1000194.

19. Mullen TE, Marzluff WF: Degradation of histone mRNA requires oligouridylation followed by decapping and simultaneous degradation of the mRNA both 5' to 3' and 3' to 5'. Genes Dev 2008, 22:50-65.

20. Rissland OS, Norbury CJ: Decapping is preceded by 3' uridylation in a novel pathway of bulk mRNA turnover. Nat Struct Mol Biol, in press.

21. LaCava J, Houseley J, Saveanu C, Petfalski E, Thompson E, Jacquier A, Tollervey D: RNA degradation by the exosome is promoted by a nuclear polyadenylation complex. Cell 2005 , 121:713-724.

22. Joachims M, Van Breugel PC, Lloyd RE: Cleavage of poly(A)binding protein by enterovirus proteases concurrent with inhibition of translation in vitro. J Virol 1999, 73:718-727.

23. Kuyumcu-Martinez M, Belliot G, Sosnovtsev SV, Chang KO, Green KY, Lloyd RE: Calicivirus 3C-like proteinase inhibits cellular translation by cleavage of poly(A)-binding protein. J Virol 2004, 78:8172-8182.

24. Alvarez E, Castelló A, Menéndez-Arias L, Carrasco L: HIV protease cleaves poly(A)-binding protein. Biochem J 2006, 396: 219-226.

25. Harb M, Becker MM, Vitour D, Baron $\mathrm{CH}$, Vende P, Brown SC, Bolte S, Arold ST, Poncet D: Nuclear localization of cytoplasmic poly(A)-binding protein upon rotavirus infection involves the interaction of NSP3 with elF4G and RoXaN. J Virol 2008, 82:11283-11293.

26. Ilkow CS, Mancinelli V, Beatch MD, Hobman TC: Rubella virus capsid protein interacts with poly(a)-binding protein and inhibits translation. J Virol 2008, 82:4284-4294.

27. Polacek $C$, Friebe $P$, Harris E: $\operatorname{Poly}(\mathbf{A})$-binding protein binds to the non-polyadenylated 3' untranslated region of dengue virus and modulates translation efficiency. J Gen Virol 2009, 90:687-692

Published: 11 August 2009

doi:10.1186/gb-2009-10-8-234

(c) 2009 BioMed Central Ltd 